receiving copper sulphate contained less than 0.5 p.p.m. of molybdenum. Plants receiving nitrogenphosphate potassium plus molybdenum (5 p.p.m. of soil) contained 24 p.p.m., rising to 38 p.p.m. where 20 p.p.m. of molybdenum was applied to the soil. This instance of molybdenum deficiency in organic soil appears to be unusual, as hitherto such conditions have been usually associated with toxicity of this element ${ }^{2}$.

Excess. Instances of a syndrome occurring in cattle associated with unthriftiness, stunted growth in young animals and rough, staring, depigmented coats have been investigated in a number of areas. The coats of red-coloured animals sometimes assume a faded yellow appearance, while although scouring is not a constant feature it has been observed in a fow cases. Symptoms are generally similar to those described by other workers ${ }^{2,4}$ as being due to a conditioned copper deficiency. Copper dosage is specific as a control. While analysis of herbage samples where affected cattle are depastured have shown normal copper values, that is, greater than 7 p.p.m. (dry matter), the molybdenum content ranged from 5 to 20 p.p.m. On the basis of data quoted elsewhere ${ }^{2}$ in connexion with a similar condition in livestock, the above figures are well within the range which represents a moderate excess, the accepted normal for herbage being in the range 1-3 p.p.m. We have also verified the latter figures for normal pasture. It seems reasonable to conclude that the level of molybdenum reported is at least partly responsible for the occurrence of this condition in livestock. While in some of the animals examined the blood copper values tended to be low, that is, of the order of $0.3 \mathrm{mgm}$./litre, this was by no means a constant finding. Hæmoglobin-levels were normal, and hæmatological studies presented no evidence of anæmia. In view of other findings ${ }^{5}$ where an association has been established between defects in bone building in cattle showing symptoms of copperdeficiency and the effects of molybdenum on the utilization of copper and indirectly of phosphorus, it is of considerable interest that we have found the condition reported to be invariably associated with herbage of high calcium content and often of wide calcium to phosphorus ratios, while in several instances blood determinations have shown that deficiencies of both copper and phosphorus co-existed in the same animal.

While this condition occurs frequently on organic soils very similar to those described ${ }^{2}$ as being associated with 'peat scours' and other nutritional troubles in livestock, in our investigations it has in addition been found to occur on what are otherwise fertile loams of limestone derivation and typical of important and widely distributed soil series. It may be of further interest that, where the worst excesses have been reported on these mineral soils, the phosphate-level of the latter has been satisfactory. This corresponds with the experience elsewhere ${ }^{6}$, as phosphate has been shown to increase molybdenum uptake and also agrees with our pot-culture findings reported above. In two instances also, basic slag used as a fertilizer accentuated the trouble, this being attributable not only to the phosphorus effect but also to the fact that basic slag contains appreciable quantities of molybdenum.

The total molybdenum content of the soils examined, with one exception so far, and representing both the deficiency and excess conditions, was normal, with a range of $0 \cdot 42-3 \cdot 50$ p.p.m. In one soil a level of 20 p.p.m. has been found. It was found that where response to molybdenum was obtained, the values were less than 0.12 p.p.m. available molybdenum, the latter being determined by the oxalate method, whereas in the soils where the herbage contained excess quantities the soil values were very considerably higher, varying from $0 \cdot 30$ to 0.57 p.p.m. of available molybdenum. Where deficiencies have been identified, the $p H$ of the soils varied from 4.9 to 5.5 ; that in soils associated with this nutritional disorder and excess molybdenum in the herbage have ranged from $p H \mathbf{H} \cdot 5$ to $7 \cdot 8$. This corresponds with other observations ${ }^{7,8}$ which show that the availability of molybdenum is greatest within the latter $p \mathrm{H}$ range. It may be of further interest also that, when a fertile soil which contained a total of 0.86 p.p.m. of molybdenum and had a $p H$ of $7 \cdot 8$ was used in a pot experiment with $T$. subterraneum, the latter contained 14 p.p.m. of molybdenum, whereas an acid soil of higher total molybdenum content $(3.5$ p.p.m.) gave a value of 0.5 p.p.m.

It is apparent that this work raises many interesting points in connexion with the treatment of pasture. Investigations are proceeding and will be reported more fully elsewhere.

[March 3.

${ }^{1}$ Anderson, A. J., J. Aust. Inst. Agric. Sci., 8 (1942). Arnon, D. I., and Stout, P. R., Plant Physiol., 14 (1939).

${ }^{2}$ Cunningham, I. J., Proc. Spac. Confr. Plant and Anim. Nutrit. Aust. (1951).

${ }^{3}$ Ferguson et al., J. Agric. Sci., 33 (1943).

- Jamieson, S., and Allcroft, R., Brit. J. Nutrit., 4, 16 (1950).

5 Davis, J. K., Contr. No. 5, McCollum Pratt Inst. (1951).

- Stout, P. R., et al., Plant and Soil, 3 (1951).

" Evans et al., Soil Sci., 71, No. 2 (1951)

Piper, C. S., and Beckwith, R. S., Proc. Spec. Confr. l'lant and Anim. Nutrit. Aust. (1951).

\section{PRINCIPLES AND APPLICATIONS OF ION EXCHANGE}

A SYMPOSIUM on the "Principles and ApplicaA tions of Ion Exchange" was held at the University of Manchester on March 27 under the joint sponsorship of the Royal Institute of Chemistry, the Chemical Society, the Society of Chemical Industry and the Institute of Petroleum. In addition to the papers contributed, an exhibition of apparatus, with demonstrations, was on view.

In the morning session, on theoretical aspects (chairman, Prof. C. W. Davies, University College of Wales, Aberystwyth), Dr. K. W. Pepper (Chemical Research Laboratories, Teddington) gave the first paper, on "Chemical Structure and Physical Characteristics of Ion-Exchange Materials". He illustrated the concept of ion-exchange resins as insoluble acids and bases by reference to certain aspects of their reactions-for example, equivalence of exchange, reversibility and accessibility of functional groups as exchange sites. After describing the preparation and chemical structure of a number of resins in some detail, particularly the most chemically stable crosslinked polystyrene type, Dr. Pepper gave an account of the characteristic behaviour of the principal types of cation and anion exchangers and indicated the of cation and anion exchangers and ing of the polymeric framework on swelling, rates of exchange and equilibria.

In his paper on "Physical Chemistry of IonExchange Equilibria and Kinetics", Dr. J. A. Kitchener (Imperial College of Science and Tech- 
nology, London) pointed out that equilibria and kinetics are relevant to practical use of resins since they govern selectivity and speed of operation. Three principal theoretical treatments, complementary rather than contradictory, have been tried involving, respectively, the law of mass action, Donnan membrane equilibrium, and ion exchange as an interaction between individual ions and charged sites. It has become clear that an adequate treatment must make allowance for the work of swelling and particularly for ionic interactions in the resin phase, a matter presenting considerable theoretical difficulty. The kinetics of ion exchange can be broadly explained by a diffusion mechanism; but a detailed quantitative theory in terms of resin structure has not yet been developed.

Dr. E. Glueckauf (Atomic Energy Research Establishment, Harwell) then discussed "Factors Affecting the Operation of Exchange Columns", indicating that while some separations depend on the separating power of the resin, others depend on a cornplexing produced in the aqueous phase. Resins with higher cross-linking are more suitable for the first type, but a lower cross-linking is better for the second. He referred to the factors, in column operation, which affect the sharpness of chromatographic bands, and distinguished between three different modes of column operation: removal of impurities (as in water softening), displacement chromatography, and development chromatography. The first two lead to fast separations and are preferable for industrial applications, but there is no complete separation into isolated bands. This only happens with the third mode, which lends itself to analytical uses. Dr. Glueckauf mentioned a recent innovation of 'gradient development' which employs an eluting solution of constantly increasing strength.

In the discussion, Dr. Kitchener, replying to Mr. J. A. Dukes, stated that he had referred to the hydrated ion when speaking of the effect of ionic size on exchange kinetics. Mr. D. K. Hale commented upon the large variations in the mass law product which can accompany variations in cationic composition of the resin and quoted supporting results of the cation pair $\mathrm{N}\left(\mathrm{C}_{2} \mathrm{H}_{5}\right)_{4}{ }^{+}$and $\mathrm{H}^{+}$, for a sulphonated cross-linked polystyrene. Treatment of the resin with an excess of $\mathrm{N}\left(\mathrm{C}_{2} \mathrm{H}_{5}\right)_{4} \mathrm{OH}$ causes an irreversible structure-change consistent with a decrease in cross-linking. Mr. Hale also mentioned difficulties which may be encountered when performing chromatographic separations with resins of low cross-linking, and stated that surface sulphonated polystyrene resins may help in difficult separations. Dr. Glueckauf reported experiments by Dr. Barker demonstrating the charged double layer, most pronounced in very dilute solutions, surrounding resin particles. Due to electrostatic repulsion, friction between resin particles is reduced, as shown by the angle of repose of a tilted bed. The effect is not shown by silver resinates.

Dr. D. D. Pratt (director, Chemical Research Laboratories, 'Teddington) opened the afternoon session on practical applications. In the first paper, on "Practical Applications of Ion Exchange in Water Treatment", Dr. F. I. Akeroyd (Permutit Co., Ltd.) reviewed the various types of ion-exchange materials, their applications in a number of water treatments, and plant and control instruments. $\mathrm{He}$ described the deionization of water and discussed the classical two-bed system, the more recent mixed-bed method, and the newer anion-exchange resins which can remove silica and other very weak acids. Dr. J. Hope (Charles Lennig and Co., Ltd.), speaking on "Other Applications", surveyed the uses of ionexchange materials in the purification of formaldehyde and glycerine, in processing beet sugar, and in the pharmaceutical industry (for example, manufacture of streptomycin). $\mathrm{He}$ also described interesting therapeutic applications, such as the treatment of peptic ulcers, heart disease, cirrhosis of the liver, and the estimation of gastric acidity.

In the last paper of the session, on "Laboratory Applications", Dr. J. E. Duncan (Harwell) dealt with three general uses of ion exchange. The first is as adsorbates - for example, in chromatographic separation of ions by direct elution with acids and alkalis, elution with complexing agents, separation of nonelectrolytes from electrolytes and separation of trace amounts; such procedures are of interest in both preparative and analytical chemistry and, in the latter case, ion-exchange is easily adapted by a radio-activation procedure and by the isotope dilution method. 'The other two uses are as laboratory reagents for titration, buffering and catalysis, and as a physico-chemical technique for the determination of ionic charge, ionic concentration, ionic size and molecular size (using dry ion exchangers as molecular sieves). In the last four applications mentioned, a detailed knowledge of the physico-chemical behaviour of the ion exchange is usually necessary.

During the discussion, Dr. B. A. J. Lister briefly described a study of the ionic size, charge and degree of formation of cation complexes in the case of zirconium, and in answer to Mr. E. Foley stated that the hydrolysis scheme for zirconium is not reversible and that hafnium may accompany zirconium in complexes. Dr. Duncan, in reply to Mr. Stevenson, quoted work by Kressman and Kitchener and others on the use of ion exchangers to determine the size of dye molecules. Mr. N. A. Hurt put a question on the removal of ammonia by cation exchangers and its effect on their capacity in water treatment. Dr. Akeroyd stated that 95 per cent of the ammonia could be removed and demonstrated the point of 'break-through' diagrammatically. A question by Dr. E. M. Meade on $\mathrm{Sn}^{2+}, \mathrm{Cr}^{2+}$ or $\mathrm{Ti}^{2+}$ resins as reductors or basic resins in the $\mathrm{CrO}_{4}{ }^{2-}$ or $\mathrm{MnO}_{4}{ }^{2-}$ form for oxidations was deferred to the evening session, in view of the comments to be made by Dr. Fairbrother. Dr. Pepper summarized applications of ion exchange in organic chemistry.

Further developments of ion-exchange materials were discussed at the evening session (chairman, Dr. F. Fairbrother, University of Manchester). Dr. R. E. Kressman (Permutit Co., Ltd.), in his paper on "Ion-Exchange Resin Membranes", described continuous sheets and rods made from both cation and anion exchangers, normally produced in granular form, and, in particular, described electrodes made from a sulphonated phenol type of resin. As expected on theoretical grounds, when these membranes separate two pure solutions, identical except in concentration, E.M.F.'s are set up which, within certain concentration limits, are predicted by the Nernst equation $E=(R T / F) \log a_{1} / a_{2}$. After discussing the results obtained-for example, with mixtures of salts, or pure solutions of salts having a common cation and different anions-Dr. Kressman concluded from his work that, within certain limits, the electrodes are useful for measuring the activities of ions in solution of salts, provided they contain one cationic species only. 
Dr. Fairbrother next gave a review of the work of Prof. H. G. Cassidy (Yale) on electron exchange polymers as oxidation-reduction systems. Quoting a letter from Prof. Cassidy, he stated that the material described ( $J$. Amer. Chem. Soc.) was largely dimer and trimer, believed to be produced by a chainterminating type of reaction of the Diels-Alder class. In more recent work, yet to be published, high polymer has readily been obtained by acetylating the monomer first, polymerizing in the presence of benzoyl peroxide, and then hydrolysing to yield polyvinyl hydroquinone which can be oxidized and reduced. The titration curve obtained was 'smeared out' in that it showed a greater slope than that of pure hydroquinone. This has been ascribed to the statistical effect of having many oxidizable groups with slightly different energies (due to their fixed location on the chains relative to other groups). Several co-polymers have been made and also crosslinked polymers to bead form.

A written contribution from Prof. M. G. Evans (University of Manchester) on possible interesting future developments with such resins was then quoted by Dr. Fairbrother. Prof. Evans likened the 'smearing out' effect to that obtained on titration of polyelectrolytes of acid or basic resins, and arises from the same causes. He pointed out that the fixing of the centres may increase the concentration of the semiquinone forms and recalled the early work of Knorr, who found that oxidation-reduction systems adsorbed on protein and cellulose structures appeared to lead to more stable semiquinones. The possibility is opened up of studying, in more detail than previously possible, the specific reactions of such entities, and it is not to be forgotten that the attachment of prosthetic groups to the protein moiety may lead to similar effects. In writing of the relative positions of the groups, Prof. Evans also referred to the possibility of studying co-operative effects, saying that if one could synthesize a resin of which the oxidation-reduction levels formed a band characteristic of the whole structure, one could imagine a process occurring at one end of the structure influencing a coupled oxidation-reduction at a considerable distance away, an interesting possibility which may arise in biological systems. Finally, he commented that the juxtaposition of two reacting centres might speed up oxidation-reduction reactions involving the simultaneous transfer of two electrons, which are normally slow, and there might be an effect similar to that of catalyst structure where a match of pattern becomes important.

Prof. Davies then briefly summarized the applications that had been discussed and pointed out that they ranged over the whole field of the design and utilization of synthetic high polymers for their chemical properties. The term 'ion exchange' has already been outgrown, and many new developments may be anticipated; electron-exchange resins are a subject of interest, and so are specially selective resins, designed to pick out one substance from a high concentration of other ions. Also, the advantage of a column procedure in pushing an equilibrium to completion can be exploited in directions other than in ion exchange- for example, in dissolving sparingly soluble salts or in the chemistry of comparatively stable complex compounds. The possibility of designing materials in which the number, kind and spacing of reactive groups can be predetermined offers a range of models to the theoretical colloid chemist and to the biochemist, who may find the catalytic properties of such materials to be of special interest. Finally, Prof. Davies said, the behaviour and use of ion exchangers in non-aqueous solvents is a subject overdue for development, and may result in laboratory applications of quite a novel kind.

In the concluding discussion Dr. Glueckauf stated that other work by Dr. Barker corroborated the findings of Dr. Kressman on ion-exchange membrane potentials of alkali solutions. Deviations from the theoretically expected potential slope, as yet unexplained, were, however, observed for calcium chloride solutions. Dr. J. D. Bu'Losk, referring to Prof. Cassidy's work and Prof. Evans's speculations on possible biological analogues of electron exchange polymers, directed attention to naturally occurring materials of this type. He cited, for example, melanin, an animal pigment which is a cross-linked polymer of indole-5:6-quinone, individual units of which should have different redox potentials, the overall effect being a smoothing-out of the oxidationreduction curve of the polymer. Natural melanin is bound to protein, forming granules associated with various enzymatic activities. Dr. Bu'Lock suggested that one function of melanin and similar natural products is to provide a suitable environment for the operation of enzymes by a kind of redox buffer mechanism.

\section{INTERNATIONAL SCIENTIFIC RADIO UNION}

$T$ HE tenth General Assembly of the Union Radio Scientifique Internationale (URSI) will be held in Sydney, Australia, during August 11-23. Of the previous nine meetings of the Union, all have been held in Europe except the second, which took place in Washington in 1927. At the previous General Assembly of the Union, held in Zurich in 1950, Sir Edward Appleton was elected president of the Union and also its delegate to the parent body-the International Council of Scientific Unions. The other officers of the Union appointed at Zurich were: Dr. J. H. Dellinger (U.S.A.), Father P. Lejay (France) and Dr. D. F. Martyn (Australia) as vice-presidents, Prof. Ch. Manneback (Belgium) as treasurer, and Eng. E. Herbays as secretary of the Union, with headquarters in Brussels.

The scientific work of the Union is organized under seven commissions, the titles and presidents of which are as follows: (1) Measurement and Standardization, Dr. J. H. Dellinger (United States); (2) Troposphere and Wave Propagation, Dr. C. R. Burrows (United States) ; (3) Ionosphere and Wave Propagation, Sir Edward Appleton (Great Britain); (4) Terrestrial Atmospherics, Prof. H. Norinder (Sweden); (5) Radio-Astronomy, Dr. D. F. Martyn (Australia); (6) Waves and Circuits, Prof. B. van der Pol (Director, C.C.I.R.); (7) Electronics, Dr. G. Lehmann (France).

The British National Committee for Scientific Radio is organized under the auspices of the Royal Society as a constituent member body of the Union Radio Scientifique Internationale. This Committee has nominated the following British delegation to the forthcoming general assembly, the numbers of the commissions to which they are specially responsible being indicated in brackets: Sir Edward Appleton (president), Dr. W. J. G. Beynon (3), Mr. J. A. 